Edwin A. M. Gale

\title{
The myth of the metabolic syndrome
}

Received: 23 June 2005 / Accepted: 23 June 2005 / Published online: 16 July 2005

(C) Springer-Verlag 2005

\section{Once upon a time}

Once upon a time, no-one knew what diabetes was. No-one doubted that someone with typical symptoms and a very high blood glucose had the condition, but a line had to be drawn between those who had diabetes and those who did not, and no-one could agree where to draw it. The great epidemiologist Kelly West wrote to his colleagues to ask what they were doing, and it emerged that they just drew their own lines wherever they wanted [1]. The difficulty they all faced was that blood glucose, as measured within a population, forms a continuous gradient between health and disease. Any cut-off between the two will therefore be somewhat arbitrary.

A line still had to be drawn, however, and there had to be a reason for drawing it. The best way to define diabetes would be according to its cause, but they didn't know what caused it, and neither do we. All they could do was to measure blood glucose. So they decided to give an oral glucose tolerance test to a whole lot of people - as it turns out, the Pima Indians of Arizona and the Civil Servants of London - and see what happened to them. They found that those with overt diabetes still had diabetes 10 years later, while those at the other end of the scale were still mostly non-diabetic. In between, matters became more complicated, for some in the middle group kept on going until they got clinical diabetes, some went back to normal, and others again stayed right where they were. One big problem was

E. A. M. Gale $(\bowtie)$

Diabetes and Metabolism,

Medical School Unit,

Southmead Hospital,

University of Bristol,

Southmead Road,

Bristol, BS10 5NB, UK

e-mail: Edwin.Gale $@$ bristol.ac.uk

Tel.: +44-117-9595337

Fax: +44-117-9595336 that the oral glucose tolerance test is not good for drawing lines with, for this gold standard measure has a large coefficient of variation around the 2-h value used to discriminate health from non-health.

And so the wise men decided that the important thing you need to know about blood glucose is how high it has to be before it harms your blood vessels. Here they came up with an answer: if your blood glucose goes up a little, this is bad for your arteries, but if it goes up a lot your small blood vessels will suffer as well. It didn't matter if you were a Pima in Arizona or worked in an office in London; the same blood glucose levels were equally bad for you. So all the world agreed that there was diabetes, non-diabetes and something in between [2], and everyone lived happily until the people in America decided that they wanted to draw the line another way [3]. This meant that some people had diabetes when they were in Europe, but not when they went to America, and the in-between groups looked quite a lot different; but people soon learned to live with the fact that we now had two different ways of defining diabetes and, of course, two scales for measuring plasma glucose.

The moral of this story is that it is very difficult to draw a line across a set of continuously distributed data to provide a yes or no answer. A line called vascular disease had been drawn across another line called blood glucose, but blood glucose changes as we grow older, and so do adiposity, blood pressure and lipids; all of which are continuously distributed variables that also relate to heart disease. Since it is not easy to draw a line for diabetes, how much more difficult would it be to draw lines for all of these variables in combination? Lines may begin as statistical artefacts, but they soon take on a life of their own. People treat them like lines on a map: sooner or later, someone will start to dispute them. This, as we have seen, was what happened with the definition of diabetes, a game played in the two dimensions of glucose and vascular disease. And so our story comes to the metabolic syndrome, a more complicated game, which is played in many dimensions at once. 


\section{The yellow brick road}

As we all know, diabetes accelerates arterial disease. It also brings unwelcome friends to the party: obesity, resistance to the actions of insulin and high blood pressure. Harry Himsworth knew this 70 years ago [4], and these features were incorporated in the first description of type 1 and type 2 diabetes in 1951 [5]. Then came the cholesterol revolution, and lipids were recognised as yet another fellow traveller on the road from diabetes to the heart. Studies of the natural history of heart disease showed that each risk factor stacks on top of all the others, that people with type 2 diabetes are generally exposed to higher risk than the rest of the population, and that they die sooner when coronary disease becomes apparent. Not surprisingly, clinical trials went on to demonstrate that the relative benefits of treating lipid levels and blood pressure are greatest in this high-risk group. As a result, we are well aware that it is important to treat blood glucose, lipids and blood pressure simultaneously, and there is overwhelming evidence to support us doing so aggressively. So what do we gain by lumping these things together under the name of the metabolic syndrome?

\section{The syndrome without a name}

By common consent, the paper presented by Gerald Reaven in 1988 represented an important new departure [6]. Reaven took the triad of diabetes, hyperlipidaemia and hypertension, and proposed that insulin resistance was the common progenitor of all three. The big contributionappropriately so for a debate that has since assumed theological dimensions - was to take a trinity of clinical features and unite them within a single all-encompassing entity. Or, to vary our cosmology, we can picture Reaven's syndrome as a world carried on four elephants (with the inclusion of obesity), each of which stands upon the giant turtle of insulin resistance. But what does the turtle stand upon? A question not to be asked. The new concept had immediate intuitive appeal but defied precise definition, and investigators rushed in to explore the new territory. First came the physiologists, whose interest lies in the mechanisms that might link insulin resistance to metabolism, and metabolism to vascular disease. Next came the epidemiologists, intent upon causation, association, risk assessment and classification, all mediated via statistical modelling derived from a rapidly spawning mass of not easily comparable studies. Then came the clinicians, greatly impressed by the energy and wisdom of their academic colleagues but unsure what difference it made to the way they looked after their patients. And behind the puzzled clinician we find the pharmaceutical companies, equipped with a solution - insulin sensitisers - for which they needed a corresponding problem. Then came the big medical organisations, each seeking to plant its own flag in the new territory and draw fresh lines on the map. And all of these groups wanted a different metabolic syndrome. The physiologists wanted a useful hypothesis, the epi- demiologists wanted to define it, public health bodies wanted to make a disease out of it, and the companies wanted to treat it.

We now have a problem that defies definition, and competing groups of investigators who each want to define it differently. All that is needed is a suitably mystical concept for them to argue about, and such a concept exists: it is called insulin resistance. What is insulin resistance? The answer to this question has more heads than a hydra, for insulin acts on different tissues in many different ways, insulin resistance can only be defined in terms of the technique used to measure it, and each technique can only examine one aspect of insulin action. Insulin-mediated glucose disposal has, by convention, become the gold standard measure of insulin resistance, and other measures, such as fasting insulin levels or HOMA, are validated against it. Given that clamp techniques are labour-intensive and hospital-based, simpler measures such as these are widely employed in population-based studies to provide a surrogate estimate of a surrogate measure of an elusive biological reality. Our definition of insulin resistance, like that of diabetes itself, is a convention based on what can be measured, and measured with admittedly imperfect techniques. Conventions are, of course, needed, for we could scarcely function without them, but only until such time as something better comes to take their place. The menu is not the meal, nor should we attempt to eat it.

\section{Prince or frog?}

No-one doubts that the human phenotype adapts to an affluent environment in a number of time-dependent and characteristic ways [7]. The question at issue is whether we should consider the metabolic syndrome as a distinct clinical entity - in other words, something that we can diagnose - or as a loosely associated cluster of consequences of a process that affects the population as a whole. Considered as a process, we can readily imagine how this might be filtered through individuals and populations that differ not only in their genes, but also in the way in which these genes will be expressed in response to complex and varying environmental pressures, which are themselves mediated by the subtleties of human behaviour. From this perspective, the anticipated spectrum of change will be as varied and diverse as the human race itself, and will never be usefully described by a single formula. The alternative point of view, more often assumed than stated, is that there is a distinct clinical entity known as the metabolic syndrome. If such an entity exists, we might reasonably expect to be able to identify its causes and the mechanisms by which these converge upon a defined set of clinical endpoints. We might expect this description to enable us to distinguish clearly between those who are affected and the remainder of the population, i.e. to provide a means of diagnosis. And, having reached a diagnosis, we might then expect this to prove useful in terms of prognosis and specific therapy targeted at prevention of the predicted outcomes. 
It emerges that not one of these conditions has been satisfied. This observation forms the core of a lengthy critical statement presented in this issue of Diabetologia and the simultaneously published issue of Diabetes Care, a statement that, by remarkable historical precedent, has been drawn up jointly by representatives of the American Diabetes Association and our own European Association for the Study of Diabetes [8]. The statement asks a number of questions. It asks if current definitions of the metabolic syndrome are useful for diagnostic purposes. It asks whether the metabolic syndrome is useful in predicting risk, and whether this exercise provides any evidence that the whole is greater than the sum of the parts. It asks whether definition of the syndrome leads to treatment that differs in any way from that already on offer for its individual components. Additional questions are then suggested, questions that will need to be answered before the debate can be taken much further. So let us consider some possible answers.

\section{Castles in the sky}

Classification of the metabolic syndrome is an activity that has almost surpassed satire. The World Health Organization (WHO) defined it in terms of major criteria - insulin resistance, diabetes or glucose intolerance/impaired fasting glucose - plus secondary criteria, i.e. the familiar triad of hypertension, hyperlipidaemia and central obesity, with the addition of microalbuminuria, which seems to have wandered in by mistake [2]. Two years later, the Expert Panel of the National Cholesterol Education Program's Adult Treatment Panel (ATP) III waded in with a new diktat that boldly discarded insulin resistance- the central pillar of the preceding definition - and opted for obesity, raised lipids, raised blood pressure and raised fasting glucose [9]. By combining these variables you can reach a diagnosis of the metabolic syndrome in 11 different ways for the WHO and 16 for the ATPIII. It would be futile to anticipate that these classifications would seek to define the component elements of the syndrome in a consistent way, for it would hardly be worth convening a committee that agreed with the criteria laid down by previous committees.

As Margaret Thatcher once said, 'when people can't agree about something, they reach a consensus', and another consensus was clearly needed. And so a major conference was held this year, at which it was proposed that the syndrome should be defined in terms of blood pressure, lipids and a fasting plasma glucose of $5.6 \mathrm{mmol} / 1$ [10]. Obesity is clearly a key element of the syndrome, but it was now recognised that different standards for waist measurement are needed for different ethnic groups. This concession to the realities of human diversity means that we no longer have a universal definition of the syndrome. As for clinicians, these might, like Yogi Berra, be forgiven for thinking that the new definition is 'déjà vu all over again'. The implications for public health are staggering, for we have created a new disease that affects some $25-30 \%$ of the population [11]. And once you have created a disease, someone will soon find it necessary to treat it.

\section{Blind men explore an elephant}

The metabolic syndrome is defined by its ability to predict heart disease and is, therefore, a matter of derivation and interpretation of statistical models. The term 'prediction', as used by medical investigators, should always be considered with caution. The word itself entered the English language as a technical term borrowed from the science of astrology [12], and acquired respectability from the natural sciences. For example, the physicists who constructed the atom bomb calculated that an explosion would result when a critical mass of radioactive material was brought together. Something never previously envisaged was predicted by logical inference from what was already known, and this achievement was possible because the underlying process was clearly understood. Although epidemiologists use the same word, they mean something rather different. Adolphe Quetelet, the Belgian father of population statistics, could predict how many citizens of Paris would commit suicide in the coming year, and by what means they would achieve it [13]. This required no insight at all into the causes of suicide; it was a purely empirical extrapolation of existing trends. The retrospective nature of such estimates would be better conveyed by the term 'postdiction' (Fig. 1).

Postdiction apart, the added temptation for those engaged in this type of analysis is to include more variables. Mediaeval scholars are said to have debated how many angels could dance on the head of a pin, and the modern equivalent is the attempt to add more features to the metabolic syndrome. This easily becomes circular, for the fact that additional variables help predict (postdict) clinical outcomes is taken as confirmation of their importance. An

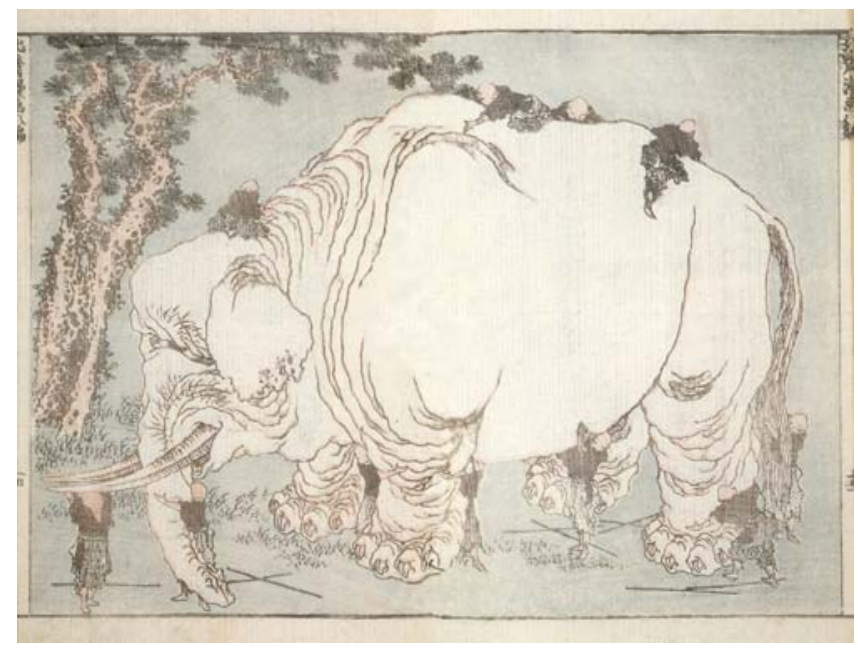

Fig. 1 Eleven blind Buddhist monks examine an elephant but fail to agree upon a definition. An illustration of the old fable by the Japanese artist Hokusai Katsushika (1760-1849) 
example may help to show how misleading this can be. If a man runs past my window, waving a stick and shouting, I might guess that a riot is in the making and claim some credit for predicting it. In contrast, I would not be considered a prophet if I drew the same conclusion after 50 angry men had stampeded past. The first men to arrive suggest that a riot is in the making; the remainder simply add strength to this conclusion. Too many studies have identified one member of the crowd, demonstrated that the presence of this individual is indicative of trouble in store, and then claimed a leadership position for him. This individual is, indeed, highly predictive of a riot, but only because he already forms part of it.

The riot principle does help in other ways, however, for the greater the number of angry men you can count, the likelier it is that a riot will ensue (or is already taking place). It will also help if you can gauge how angry they are. Clinicians do this all the time, and the main principle of clinical risk estimation is not to discard useful information. When I sit in the clinic, I need to know the age, sex, smoking habits and family history of my patient, and whether he or she has diabetes or established heart disease. I then measure blood pressure, glucose and lipids, but I do not convert the results into yes or no answers; instead, I note a slightly elevated blood pressure and grossly elevated triglycerides. The label of metabolic syndrome may or not be applicable, but would not influence my management in any way whatsoever, and is therefore irrelevant. And if I wanted to estimate risk in a population rather than an individual, I would want to use continuous rather than dichotomous variables, since they give me more information. This is why other scales of cardiovascular risk generally outperform the metabolic syndrome [8]. Finally, we can note that the metabolic syndrome score is so heavily weighted towards diabetes and established arterial disease that it loses most of its prognostic significance once these obvious risk markers have been eliminated [8].

\section{Happily ever after}

The debate about the metabolic syndrome is littered with logical traps. The first and most fundamental is known as reification, which is what happens when an abstraction is mistaken for a thing. Richard Asher, who gave the Munchausen syndrome its name, subsequently warned that the effect of doing so had been to convert a clinical observation into an entity, which - to his horror - soon became the subject of academic study and debate. 'When christening a baby', he said, 'we wait for the child to be born and then we find a name for it. When christening a disease we sometimes wait for the name to be born and then we try to find a disease to suit it' [14]. Once the metabolic syndrome had been named, it was assumed to exist. If it exists, we must be able to define it. If clinicians can define it, it must be a disease. And if it is a disease, we must, of course, be ready to treat it. Such are the consequences of giving an abstract entity a name. Nor should we forget the commercial implications of doing so. The
International Diabetes Federation statement, for example, discusses a possible future role for the thiazolidinediones in managing the syndrome [10]. Speculation perhaps, but we may note in passing that the effect of treating the atrisk population of the Western world with the current generation of these agents would be to add some 520 million kilos of adipose tissue to the populations of Europe and North America - a truly novel means of combating the obesity epidemic.

The circular nature of many of the discussions about the metabolic syndrome should not be overlooked. Those who defined diabetes did so on the basis of its consequences for vascular disease, a useful although back-to-front way of doing things, and they used the single yardstick of glucose. In much the same way, the definition of the metabolic syndrome has been retrofitted around its implications for arterial disease. But in this case, there is no single yardstick; each variable is adjusted according to its utility in prediction. Shorn of unconsidered factors, such as cigarette smoking and family history, risk of arterial disease defines the syndrome, and the syndrome defines arterial risk, thus creating a perfect loop. This is why it is impossible to distinguish between the definition of the metabolic syndrome, the predictive power of the syndrome and its implications for treatment. They are all, by self-confirming circularity, one and the same thing.

There is no question that risk factors for heart disease cluster together. The question at issue is whether this cluster of features conceals a distinct clinical entity. A clinical entity might be said to exist if it had a distinctive cause, or a distinctive set of clinical features, or a distinctive set of consequences. We have seen that insulin resistance, important though it may be, provides an insecure foundation for the metabolic syndrome, to the extent that it is no longer considered a useful (or at least measurable) criterion. We have also seen that no single clinical feature is necessary for a diagnosis, and that current definitions are based upon a heterogeneous patchwork of overlapping variables. And, finally, there is no distinct set of consequences - the whole is not greater than the sum of the parts - and adverse outcomes can be predicted equally well or better from ad hoc measures of risk such as the Framingham score [8]. Reaven himself concludes a recent review with the statement that 'the diagnosis of the metabolic syndrome does not bring with it much in the way of pathophysiologic understanding or clinical utility' [15], and there is no reason to believe that the metabolic syndrome exists as a distinct clinical entity.

In conclusion, there is no question that a number of associated clinical features congregate in individuals who are at increased risk of heart disease. They cluster together for a reason, and it is important to seek an explanation for this at a pathophysiological level. So must we also seek to understand the interactions between arterial disease, insulin resistance, blood pressure, lipids and glucose, and the impact of these risk factors at a population level. These are the phenomena upon which our attention should be focused. The rest is for people who like fairy tales. 
Acknowledgements I thank Knut Borch-Johnsen for stimulating discussion and critique, and David Matthews for suggesting the term 'postdiction'.

\section{References}

1. West KM (1975) Substantial differences in diagnostic criteria used by diabetic experts. Diabetes 24:641-644

2. World Health Organization (1999) Definition, diagnosis, and classification of diabetes mellitus and its complications: report of a WHO consultation. Geneva, World Health Organization

3. American Diabetes Association (2000) Screening for type 2 diabetes. Diabetes Care 23(Suppl 1):20-23

4. Himsworth H (1936) Diabetes mellitus: a differentiation into insulin-sensitive and insulin-insensitive types. Lancet i:127130

5. Lister J, Nash J, Ledingham U (1951) Constitution and insulin sensitivity in diabetes mellitus. Br Med J i:376-379

6. Reaven GM (1988) Role of insulin resistance in human disease. Diabetes 37:1595-1607

7. Gale EAM (2004) The changing phenotype of the human species (affluent variety). Diabetologia 47:1339-1342
8. Kahn R, Buse J, Ferrannini E, Stern M (2005) The metabolic syndrome: time for a critical appraisal. Diabetologia DOI 10.1007/s00125-005-1876-2

9. Expert Panel on the Detection, Evaluation, and Treatment of High Blood Cholesterol in adults (2001) Executive summary of the third report of the National Cholesterol Education Program (NCEP) expert panel on detection, evaluation and treatment of high blood cholesterol in adults (Adult Treatment Panel III). JAMA 285:2486-2497

10. International Diabetes Federation (2005) The IDF consensus worldwide definition of the metabolic syndrome. Available from http://www.idf.org last accessed June 2005

11. Ford ES, Abbasi F, Reaven GM (2005) Prevalence of insulin resistance and the metabolic syndrome with alternative definitions of impaired fasting glucose. Atherosclerosis 181:143148

12. Soanes C, Stevenson A (eds) (2004) Concise Oxford English Dictionary, 11th edn. Oxford University Press, Oxford

13. Quetelet A (1835) Sur l'homme et le développement de ses facultés, ou Essai de physique sociale. Bachelier, Paris. (Reprinted in Elibron Classics Replica Edition)

14. Asher R (1959) Making Sense. Lancet ii:359-365

15. Reaven GM (2005) The metabolic syndrome: requiescat in pace. Clin Chem 51:930-931 